\title{
Comparison of Different Methods for Detection of Biofilm Formation in Staphylococcus aureus in a Tertiary Care Hospital
}

\author{
Prasanna Gupta ${ }^{1}$, Manish Kumar Diwakar ${ }^{2}$, Santosh Verma ${ }^{3}$ and Neha Srivastava ${ }^{4}$ \\ ${ }^{1}$ Department of Microbiology, NIMS Medical college, Jaipur, Rajasthan, India \\ ${ }^{2}$ Department of Microbiology, ${ }^{3}$ Department of Community Medicine, MLB Medical College, \\ Jhansi (UP), India \\ ${ }^{4}$ Department of Microbiology, Rama Medical College, Kanpur (UP), India \\ *Corresponding author
}

\section{A B S T R A C T}

\begin{tabular}{|l|}
\hline Key w or d s \\
Biofilm, Tissue \\
culture plate \\
method, Congo red \\
agar, \\
Staphylococcus \\
aureus, MRSA
\end{tabular}

Biofilm is the population of bacterial cells growing on the biotic and abiotic surfaces embedded in a matrix of extracellular polysaccharide, which facilitate the adherence of microorganism. This study is aimed to compare and evaluate different Methods for detection of Biofilm formation in Staphylococcus aureus isolates. For comparison of different methods of Biofilm production, 100 isolates of Staphylococcus aureus (50 MRSA and 50 MSSA) were taken and screened by tissue culture plate method, tube method in different condition and Congo Red Agar (CRA) plate method. Out of 100 isolates of Staphylococcus aureus, 19\% shows Strong biofilm formation, while $33 \%$ showed moderate and $48 \%$ isolates showed weak/No biofilm production by TCP Methods. In TM-Gr-1: 10\%, 28\% and 62\%; in TM-Gr-2: 10\%, 20\% and 70\%; in TM-Gr3: $16,29 \%$ and $55 \%$ isolates showed strong, moderate and weak/ No biofilm production respectively. The CRA Method showed $17 \%$ biofilm production. The TCP method was found to be most sensitive screening method for detection of biofilm formation by staphylococcus aureu. MRSA isolates were more Biofilm producers than MSSA.

\section{Introduction}

Biofilm is the population of bacterial cells growing on the biotic and abiotic surfaces embedded in a matrix of extracellular polysaccharide, which facilitate the adherence of microorganism. Staphylococci are most common organism associated with chronic infections of implanted medical devices (Astha and Amita Jain, 2012; Donlan, 2002).

The use of indwelling medical devices in clinical set up is important in the patient management in Intensive care unit as well as of critically and chronically ill patients, however bacterial colonization of implanted foreign material can cause major medical and economic sequel.

The increased use of indwelling medical devices has increased the impact of Staphylococci in clinical settings. The predominant species isolated in these infections are Staphylococcus aureus and Staphylococcus epidermidis. Biofilm formation is their major pathogenicity (Kloos et al., 1994). 
Biofilm is a group of organisms growing as a layer on a surface. Biofilm thickness can range from a single cell layer to a substantial community encased by a viscous polymeric milieu. Structural analyses have shown that in some cases unique pillar or mushroom-shaped structures can be formed by the micro-colony architecture of these dense biofilms; however, other structures do form depending on the environmental conditions (Costerton et al., 1995). Biofilm consists of multilayered cell clusters embedded in a matrix of extracellular polysaccharide, which facilitates the adherence of these microorganisms to biomedical surfaces or devices and protect them from host defence system and antimicrobial treatment (Gara et al., 2001).

Staphylococcus aureus is well-known bacteria to make biofilms on different human body surfaces as well as indwelling devices. The microbes forming the biofilm are difficult to treat in clinical settings. These isolates may or may not be resistant to anti-bacterial agents in laboratory setting, but due to difficulty in eradication of the biofilm formed on the surfaces of the devices/appliances and protection provided to the microorganism by protective covering of adhesive biomaterial (slime), it becomes difficult to treat infections caused by these organisms. An association between antibiotic resistance and biofilm production in nasal isolates of Staphylococci have been reported.

Astha and Amita Jain (2012) found in their study that there is a significant high rate of Biofilm production in nasal isolates of $S$. aureus. Various researchers (Astha, 2012; katayama et al., 2000) have observed that Antibiotic resistance among biofilm producer was significantly higher than that of Nonbiofilm producers.

Staphylococci are ubiquitous pathogens that usually produce biofilms during different infectious processes, which are generally difficult to treat. It has been estimated that about 65 per cent of the hospital acquired infections are associated with biofilm forming organism. It has been assumed that these infections are 10 to 1000 times more difficult to eliminate with an otherwise successful treatment. The mechanism for enhanced antimicrobial resistance is believed to involve alteration in gene expression leading to a phenotypic difference between the planktonic and sessile forms. The planktonic forms are more resistant as they produce exopolysaccharide, have different growth characteristics and take up nutrients and drugs differently as compared to their planktonic counterparts.

De Araujo et al., (2006) reported that biofilm producing Methicillin resistant S. epidermidis isolates from healthy individuals from the community had a higher incidence of multiresistance than biofilm non-producers from the same population. They also noticed increased incidence of multi resistance among biofilm producers compared to non-producers. It is mentioned clearly in literature that over $65 \%$ of hospital based infections caused by the biofilm producing organisms (Kloos and Bannerman, 1997). It is now well documented that biofilm are notoriously difficult to eradicate and are often resistant to systemic antibiotic therapy and removal of infected device becomes necessary (Lewis, 2001; Schwank et al., 1998; Souli et al., 1998; Astha and Amita Jain, 2012).

The detection of biofilm producer and non producer Staphylococci might help to elucidate the impact of Staphylococci in diagnosis of infections related to biomedical devices and these observations may have utility in the prevention of device related infections and better patient management (Astha and Amita Jain, 2012). There are number of phenotypic tests available to detect biofilm production by Staphylococci which 
include tissue culture plate (TCP), tube method (TM), Congo red agar (CRA) (Christensen et al., 1985; Freeman, 1989 and Mathur et al., 2006).

\section{Materials and Methods}

A total of 100 non-repetitive, clinical and carriage isolates of Staphylococcus species were investigated for the production of Biofilm by using different methods. Isolates were initially identified by standard microbiological techniques including Gram stain, catalase test, coagulase test, Mannitol fermentation and DNase test.

All cultures were maintained on Brain Heart Infusion agar (Himedia Ind Pvt Ltd.). Known reference strains of $S$. epidermidis ATCC 35984 (high slime producer), S. epidermidis ATCC 35983 (moderate slime producer) and S. epidermidis ATCC 12228 (non slime producer) obtained from Himedia Ind Pvt Ltd. were used as controls in the study (Winn et al., 2006; Mackie and McCartney practical medical microbiology. 14th ed, 2012; Koneman, 2016 and CLSI, 2017)

For comparison of different methods of Biofilm production, 100 isolates of Staphylococcus aureus (50 MRSA and 50 MSSA) were taken and screened by Tissue Culture plate Method (TCP) by using BHI broth supplemented with $2 \%$ sucrose for 24 hrs.

Three different conditions was applied in Tube Method (TM) by incubating isolates at 370C in BHI broth supplemented with $1 \%$ Glucose for $24 \mathrm{hrs}$ (Group-1), and in BHI broth supplemented with $1 \%$ sucrose for $24 \mathrm{hrs}$ (Group-2) and in BHI broth supplemented with $1 \%$ sucrose for $48 \mathrm{hrs}$ (Group-3). Congo Red Agar (CRA) Plate method was also used to detect biofilm production.

\section{Tissue culture plate method (TCP)}

The TCP assay described by Christensen et al., (1985) and Mathur et al (2006) was used in this study. For comparative study of different methods TCP method was considered as standard test for detection of biofilm formation. In this study, we had screened all isolates to check their ability to form biofilm by TCP method as described by Christensen et al., (1985) with a modification in duration of incubation which was extended to 24 hours.

Isolates from fresh agar plates were inoculated in Brain heart infusion broth media supplemented with $2 \%$ sucrose and incubated for 18 hour at $37 \mathrm{oC}$ in stationary condition and diluted 1in100 with fresh medium. Individual wells of sterile, polystyrene, 96 well-flat bottom tissue culture plates (Tarson, Kolkata, India) wells were filled with $0.2 \mathrm{ml}$ aliquots of the diluted cultures and only broth served as control to check sterility and nonspecific binding of media.

The tissue culture plates were incubated for 18 hours and 24 hours at $37^{\circ} \mathrm{C}$. After incubation content of each well was gently removed by tapping the plates. The wells were washed four times with $0.2 \mathrm{ml}$ of phosphate buffer saline (PBS pH 7.2) to remove free-floating planktonic bacteria. Biofilms formed by slime producing organisms in the tissue culture plate were fixed with sodium acetate $(2 \%)$ and stained with crystal violet $(0.1 \% \mathrm{w} / \mathrm{v})$. Excess stain was rinsed off by thorough washing with deionized water and plates were kept for drying. Slime forming adherent staphylococcal cells usually formed biofilm on all side wells and were uniformly stained with crystal violet. Optical density (OD) of the tissue culture plate was calculated by micro ELISA auto reader at wavelength of $570 \mathrm{~nm}$ (OD- $570 \mathrm{~nm}$ ). The observed OD values were considered as an index of bacteria adhering to surface and biofilm formation. 
To compensate for background absorbance, the OD value of sterile medium is subtracted from the OD of all test values. Thus, the mean OD value obtained from media control well was deducted from all the test OD values which give the Result.

\section{Interpretation of the result}

The optical density will be recorded using an ELISA reader at the wavelength of $570 \mathrm{~nm}$. The strains that gave an OD value less than 0.120 will be recorded as non-biofilm producers, those with OD values between 0.120 and 0.240 will be considered as moderate producers and those with OD values more than 0.240 will be considered as strong producers.

\section{Tube method}

A qualitative assay for biofilm formation was determined as previously described by Christensen et al (1982) with some modifications in media introduced and incubation time.

Three different conditions was applied in Tube Method (TM) by incubating isolates at 370C in BHI broth supplemented with $1 \%$ Glucose for $24 \mathrm{hrs}$ (Group-1) and in BHI broth supplemented with $1 \%$ sucrose for $24 \mathrm{hrs}$ (Group-2) and in BHI broth supplemented with $1 \%$ sucrose for $48 \mathrm{hrs}$ (Group 3).

Each group of Liquid culture media $(10 \mathrm{ml})$ was inoculated with loopful of microorganism from overnight culture plates and incubated for 24 hours and 48 hours at $37^{\circ} \mathrm{C}$. The tubes were decanted and washed with Phosphate Buffer Saline (pH 7.3), dried and then stained with crystal violet $(0.1 \%)$. Excess stain was removed and tubes were washed with deionised water. Tubes were than dried in inverted position and observed for a visible film of the stain.
Biofilm formation was considered as positive when a visible layer of crystal violet smeared the wall and bottom of the tube. Ring formation at the liquid interface is indicative of non biofilm formation. Tubes were observed for the intensity of colour adherence and the amount of biofilm formation was recorded as 0 -absent, 1-weak, 2-moderate or 3 -strong.

\section{Congo red Agar method (CRA)}

The bio film detection by Congo Red Agar was performed as per the method described by Mathur et al., (2006) for detection of biofilm a special media was prepared. The medium was composed of BHI (37 gms/L), sucrose (100 $\mathrm{gms} / \mathrm{L})$, agar no.1 (10 gms/L) and Congo red stain $(0.8 \mathrm{gms} / \mathrm{L})$. Congo red was prepared as concentrated aqueous solution by autoclaving at $121^{\circ} \mathrm{C}$ for 15 minutes. It was prepared separately from other medium constituents and then added to the agar when cooled to $55^{\circ} \mathrm{C}$. Plates were poured make let them allow to settle for about 10-15 minutes. Then the isolates were inoculate and incubated aerobically for 24 to 48 hours at $37^{\circ} \mathrm{C}$.

\section{Interpretation of the results}

Positive result was indicated by black colonies with a crystalline dry consistency. Weak producers usually remained red. A darkening of the colonies without the dry crystalline colonial morphology indicated an indeterminate result.

\section{Results and Discussion}

Out of 100 isolates of Staphylococcus aureus, 19\% (14 MRSA and 05 MSSA) shows Strong biofilm formation, while $33 \%$ showed moderate (21 MRSA and 12 MSSA) and $48 \%$ isolates (15 MRSA and 33 MSSA) showed weak/No biofilm production by Tissue Culture Plate Methods. In Tube Method-Group-1, 
$10 \%$ of $S$. aureus isolates show strong biofilm producer in which 7 were MRSA and 03 MSSA were MSSA. Similarly $28 \%$ of isolates showed moderate biofilm producer among which 20 isolates were MRSA and 08 were MSSA while $62 \%$ isolates were non biofilm producer among which 23 isolates were MRSA and 39 isolates were MSSA); in Tube Method-Group-2, 10\% (7 MRSA and 03 MSSA), 20\% (12 MRSA and 08 MSSA) and $70 \%$ (31 MRSA and 39 MSSA); in Tube Method-Group-3, 16\% (12 MRSA and 04 MSSA), 29\% (18 MRSA and 11 MSSA) and 55\% (20 MRSA and 35 MSSA); isolates showed strong, moderate and weak/ No biofilm production respectively. The CRA Method showed least and only $17 \%$ of isolates to be biofilm producer among which 12 isolates were MRSA and 5 were MSSA. Biofilm production with highly correlated with TCP method and Tube Method Group-3 but was least sensitive method.

The TCP method found to be the most effective screening method for detection of biofilm formation by Staphylococcus aureus and has the advantage of quantitative analysis of the biofilm formation by Staphylococci. MRSA isolates were more Biofilm producers than MSSA.

Biofilm production has been reported in strains of all Staphylococcus species associated with the infection of biomedical devices (Kooles et al., 1994). Investigations to understand the pathogenesis of these infections have focused upon the process of adherence of these microorganisms on these devices. Investigators have used various methods to quantify number of microorganisms adhering to surfaces (Panda et al., 2014; Donlan, 2001) We tested 100 clinical and carriage isolates of Staphylococci by three in vitro screening procedures to detect the ability of biofilm production.
In the Tissue culture plate method, by using BHI broth supplemented with sucrose; only 52 $\%$ Staphylococcus isolates show biofilm production. In modified TCP method, incubation period is extended for 24 hour. It provides a better discrimination between moderate and non-biofilm producing Staphylococci and biofilm formation.

While correlating with test tube method with the TCP test method for strong biofilm producing isolates, it was difficult to discriminated between weak and biofilm negative isolates due to the variability in observed results by different observers which was also experienced by other authors (Sasirekha et al., 2012; Maryam Rezaei, 2013) Consequently, high variability was observed and classification in biofilm producer and Non Producer was difficult by tube method. This depends upon skill of the researcher. In agreement with the previous reports, tube test cannot be recommended as general screening test to identify biofilm-producing isolate (Christensen et al., 1982). In CRA method, out of 100 staphylococcal isolates, seventeen (17\%) showed black colonies with dry crystalline morphology. These observations are entirely in disagreement with observations reported by the Maryam Rezaei et al., (2013) and A Jain et al., (2012) in which he found better sensitivity of the method. The similar finding was observed by Praharaj et al., (2013), Tayal Ruche (2015) which established that the TCP method is more reliable, sensitive and specific (Fig. 1).

Based on our results and observation us contempt to the CRA method for detection of biofilm formation for Staphylococcus isolates. Our data indicates that the TCP method is an accurate and reliable method for screening and this technique can also able to serve as a reliable quantitative tool for determining biofilm formation. 


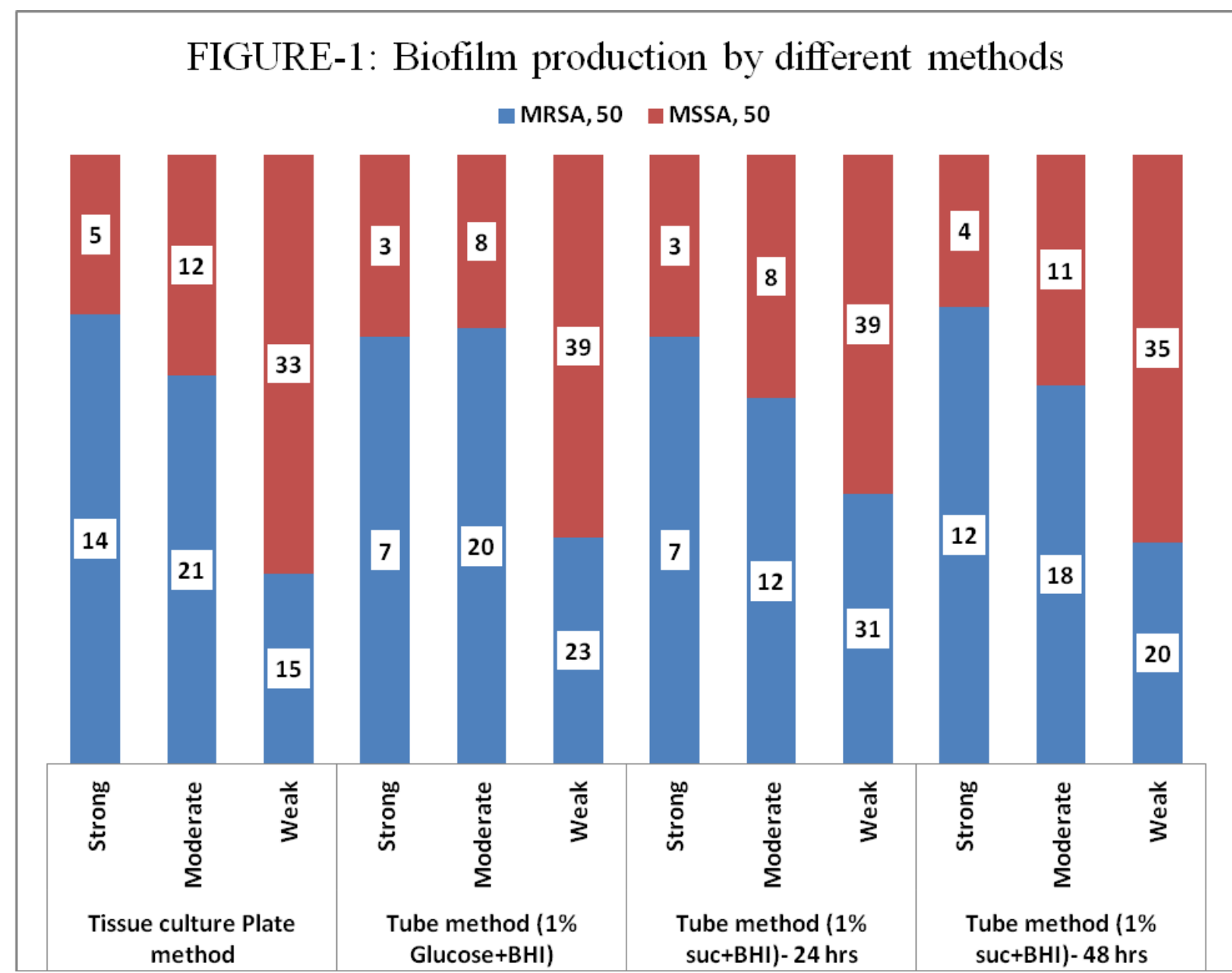

\section{References}

Astha Agarwal and Amita Jain, Association between drug resistance and production of biofilm in Staphylococci, Indian $\mathbf{J}$ Med Res. 2012 April; 135(4): 562-564.

T Mathur, S Singhal, S Khan, DJ Upadhyay, T Fatma, A Rattan, Detection of biofilm formation among the clinical isolates of Staphylococci: An evaluation of three different screening methods, Indian Journal of Medical Microbiology, (2006) 24 (1):25-9.

Adilson Oliveira, Maria de Lourdes R S Cunha, Comparison of methods for the detection of biofilm production in coagulase negative Staphylococci

Ammendolia MG, Rosa RD, Montanaro L, Arciola CR, Baldassarri L. Slime production and expression of slim- associated antigen by staphylococcal clinical isolates. J Clin Microbiol 1999; 37: 3235-8.

Arciola CR, Baldassarri L, Montanaro L. Presence of icaA and icaD genes and slime production in a collection of staphylococcal strains from catheterassociated infections. J Clin Microbiol 2001; 39: 2151-6.

Baird D. Staphylococcus: cluster-forming Gram-positive cocci. In: Collee JG, Marmion BP, Fraser AG, Simmons A, editors. Mackie and McCartney practical medical microbiology. 14th ed. New Delhi: Elsevier; 2012. p. 255.

Christensen GD, Simpson WA, Bisno AL, Beachey EH. Adherence of slimeproducing strains of Staphylococcus epidermidis to smooth surfaces. Infect Immun 1982; 37: 318-26. 
Christensen GD, Simpson WA, Younger JA, Baddour LM, Barrett FF, Melton DM, et al., Adherence of coagulase negative Staphylococci to plastic tissue cultures: a quantitative model for the adherence of Staphylococci to medical devices. J Clin Microbiol 1985; 22: 996-1006.

Christensen GD, Simpson WA, Younger JA, Baddour LM, Barrett FF, Melton DM.et al., Adherence of cogulase negative Staphylococci to plastic tissue cultures: a quantitative model for the adherence of Staphylococci to medical devices. J Clin Microbiol 1985; 22: 996-1006.

CLSI document M100-S17. Wayne Pa: CLSI; 2007. Clinical and laboratory standards institute. Performance standards for antimicrobial susceptibility testing; seventeenth informational supplement

Costerton JW, Lewandowski Z, Caldwell DE, Korber DR, Lappin-Scott HM. Microbial biofilms. Annu Rev Microbiol 1995; 49:711-45; PMID: 8561477; DOI: 10.1146/annurev.mi. 49.100195.003431.

Cramton SE, Gerke C, Schnell NF, Nichols WW, Gotz F. The intracellular adhesion (ica) locus is present in Staphylococcus aureus and is required for biofilm formation. Infect Immun 1999; 67: 5427-33.

De Araujo Gl, Coelho LR, de Carvalho CB, Maciel RM, Coronado AZ, Rozenbaum $\mathrm{R}$, et al., Commensal isolates of Methicillin Resistant Staphylococcus epidermidis are also well equipped to produce biofilm on polystyrene surfaces. J Antimicrob Chemother. 2006; 57: 85564.

Donlan RM, Murga R, Bell M, Toscano CM, Carr JH, Novicki TJ, et al., Protocol for detection of biofilms on needleless connectors attached to central venous catheters. J Clin Microbiol 2001; 39: $750-3$.
Dunne WM. Bacterial adhesion: Seen any good biofilms lately. Clin Microbiol Rev 2002;15:155-66.

Freeman DJ, Falkiner FR, Keane CT. New method for detecting slime production by coagulase negative Staphylococci. J Clin Pathol 1989; 42: 872-4.

Johannes KM, Knobloch M, Matthias A, Rohde H, Mack D. Evaluation of different methods of biofilm formation in Staphylococcus aureus. Med Microbiol Immunol 2002; 191: 101-6.

Katayama, Y., Ito, T. and Hiramatsu, K. (2000) A new class of genetic element, Staphylococcus cassette chromosome mec, encodes methicillin resistance in Staphylococcus aureus. Antimicrob Agents Chemother., 44, 1549-55.

Kloos WE, Bannerman TL. Update on clinical significance of coagulasenegative Staphylococci. Clin Microbiol Rev., 1994; 7: 117-40.

Lewis K. Riddle of biofilm resistance. Antimicrob Agents Chemother 2001; 45: 999-1007.

Ludwicka A, Switalski LM, Lundin A, Pulverer G, Wadstrom T. Bioluminescent assays for measurement of bacterial attachment to polyethylene. J Microbiol Methods 1985; 4: 169-77.

Maryam Rezaei, Rezvan Moniri, Seyed Gholam, Abbas Mousavi, Marzie Jabari Shiade. Prevalence of biofilm formation among methicillin resistance Staphylococcus aureus isolated from nasal carriers. Jundishapur J Microbiol. 2013 Aug; 6(6): e9601.

O Gara JP, Humphreys H. Staphylococcus epidermidis biofilms: importance and implications. J Med Microbiol 2001; 50: 582-7.

Panda PS, Choudhary U, Yadav S, Dube SK. Biofilm production in gram-positive isolates causing urinary tract infection in a tertiary care hospital. Indian $\mathbf{J}$ Pathol Microbiol. 2014; 57: 159-60. 
Praharaj I, Sistla S and Parija SC, 2013. Virulence factors in Clinical and Commensal isolates of Enterococcus species. Ind J Path Microbiol., 56(1): 24-30.

Raad I, Darouiche R, Hachem R, Sacilowski M, Bodey GP. Antibiotics and prevention of microbial colonization of catheter. Antimicrob Agents Chemother 1995; 39: 2397-400.

Sasirekha B, Usha MS, Amruta AJ, Ankit S, Brinda N, Divya R. Evaluation and comparison of different phenotypic tests to detect methicillin resistant Staphylococcus aureus and their biofilm production. Int J PharmTech Res. 2012; 4(2): 532-41.

Schwank S, Rajacic Z, Zimmerli W, Blaser J. Impact of bacterial biofilm formation on in vitro and in vivo activities of antibiotics. Antimicrob Agents Chemother 1998; 42: 895-8.

Souli M, Giamarellou H. Effects of Slime produced by clinical isolates of coagulase negative Staphylococci on activities of various antimicrobial agents. Antimicrob Agents Chemother., 1998; 42:939-41.

Tayal Ruchi, Baveja Sujata and De Anuradha, Original Research Article Comparison of Phenotypic Methods for the Detection of Biofilm Production in Uro-Pathogens in a Tertiary Care Hospital in India, Int.J.Curr.Microbiol. App.Sci (2015) 4(9): 840-849.

Winn WC, Jr, Koneman EW, Allen SD, Procop GW, Janda WM, Schreckenberger PC, et al., 6th ed. Baltimore (USA): Lippincott Williams and Wilkins; 2006. Koneman's color atlas and textbook of diagnostic microbiology.

Zufferey J, Rime B, Francioli P, Bille J. Simple method for rapid diagnosis of Catheter-associated infection by direct Acridine orange staining of catheter tips. J Clin Microbiol 1988; 26: 175-7.

\section{How to cite this article:}

Prasanna Gupta, Manish Kumar Diwakar, Santosh Verma and Neha Srivastava. 2018. Comparison of Different Methods for Detection of Biofilm Formation in Staphylococcus aureus in a Tertiary Care Hospital. Int.J.Curr.Microbiol.App.Sci. 7(05): 2548-2555. doi: https://doi.org/10.20546/ijcmas.2018.705.293 